\title{
Analysis of Circuit Identification for Single Ring Space Agencies
}

\author{
Fei Zhou ${ }^{1, a^{*}}$ and Guomin $\operatorname{Lin}^{1, b}$ \\ ${ }^{1}$ College of Mechanical Engineering, Xijing University, Xi'an, China \\ a743040357@qq.com, b461922530@qq.com
}

Keywords: Single-loop bodies; Circuit identification; Branch offices; Identify dead point location

\begin{abstract}
Space agency of the circuit is more complicated than the planar mechanism. Different circuits have different rotational situation in planar multi-loop mechanism. For more than four or closed form space monocyclic institutions existed. In this article, monocyclic institutions is introduced; Foundation of loop identification is illustrated; Branch of automatic identification is analyzed; A new method of recognition space loop of monocyclic mechanism and its defects have been proposed, thus each dead point position is identified. This is an important theoretical value for the further study of space ring structure.
\end{abstract}

\section{Introduction of Monocyclic Institutions}

Has four Spaces monocyclic institutions closed form refers to the input output displacement equation for four times. This kind of monocyclic institutions including space RCRCR, RSCR, RRSRR, RSRRR., RRSPR institutions, etc. Space agency of the circuit is more complicated than the planar mechanism. Only in planar multi-loop mechanism will appear different circuits with different rotational, for four or more closed form space monocyclic institutions existed [1]. When an input corresponding to multiple outputs, multiple real root, to calculate the correct distinguish which one they are all belong to the circuit, it is not an easy thing, if wrong agencies to conduct a comprehensive motion analysis, and circuit and its defect is much more difficult to identify, so has four closed form space monocyclic agency! R, 1, automatic identification of the road to the recently has not yet been solved.

\section{Foundation of Circuit Identification}

Basic Idea of Loop Identification. If $\Delta=0$ has $n$ real solution: $\theta 1, \theta 2, \theta 3 \ldots \theta n,(n>0)$, it said the input components have $\mathrm{n}$ rest position, for any of the input components rest position $\theta \mathrm{i}(\mathrm{i}=1,2, \ldots, \mathrm{n})$, its corresponding output solution only four and two solutions in both cases [2]. Through in-depth study found that the input component of any stationary position theta, I dead point distribution of the agency have seven types as follows:

$\theta \mathrm{i}$ corresponding mechanism of a dead point DP, I have two branches of the new generation in DP, as shown in Fig. 1 (a) [3]. $\theta$ i corresponding mechanism of a dead point DP, in DP has two branches of the old union, as shown in Fig. 1 (b). $\theta$ i corresponding mechanism of two dead DP1, DP2, and each had two new branch generated dead point, as shown in Fig. 1 (c) [4]. $\theta \mathrm{i}$ corresponding mechanism of two dead DP1, DP2, and each had two old dead center branch, as shown in Fig. 1 (d) [5]. $\theta \mathrm{i}$ corresponding mechanism of two dead DP1, DP2, among them at a dead point has two branches of the old union, at another dead center has two branches of the new generation, as shown in Fig. 1 (e). 


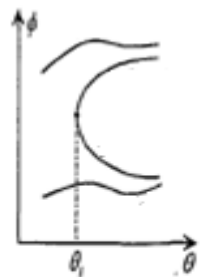

(a)

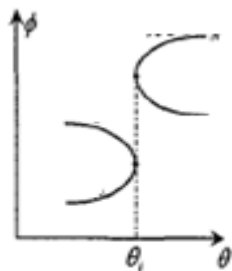

(e)

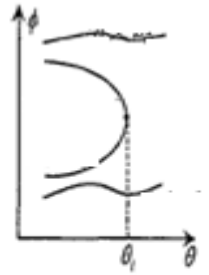

(b)

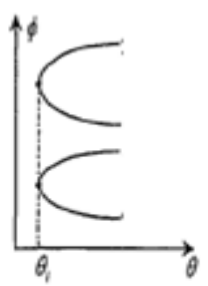

(c)

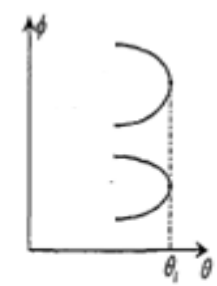

(d)

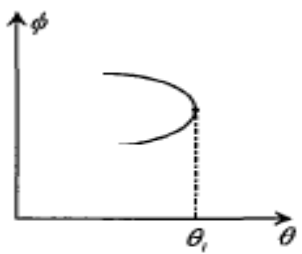

(f)

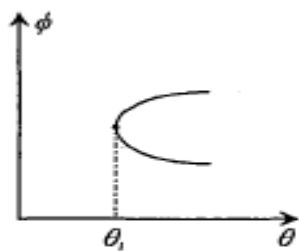

(g)

Figure 1. Dead point type diagram

Loop to identify the basic idea is: first of all find out discriminant delta $=0$ : all real solution $\theta 1<\theta 2<\theta 3<\theta 4<\ldots<\theta$ n, and then by the $\theta \mathrm{i}(\mathrm{i}=1,2, \ldots, \mathrm{n})$ and the number of the output of the corresponding solution and the numerical, determine the corresponding to the distribution of dead point, record each dead center in combination of two branches, between adjacent two dead center branch number and branch number, etc [6]., Finally based on every dead center Branch, the branch combination for loop, and according to the input link between the adjacent two stationary position of branch number and branch number, you can identify circuit defect. Criterion 1 corresponds to the dead situation as shown in Fig. 2 [7]. Criterion 2 corresponds to the dead situation as shown in Fig. 3 [8]. Criterion 3 corresponding dead center situation as shown in Fig. 4. Criterion of four corresponding dead center situation as shown in Fig. 5. The corresponding criterion of dead case as shown in Fig. 6.

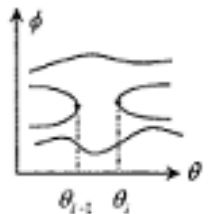

(a)

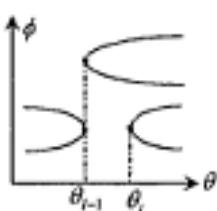

(b)

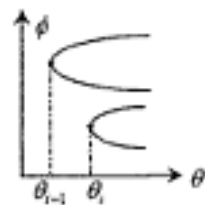

(c)

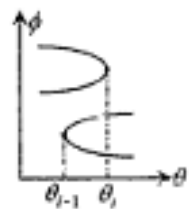

(a)

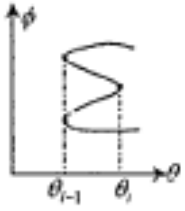

(b)
Figure 2. Crtterion 1 corresponds to the dead

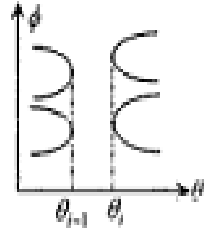

(a)

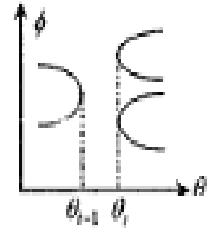

(b)
Figure 4. Crtterion 3 corresponds to the dead
Figure 3. Crtterion 2 corresponds to the dead

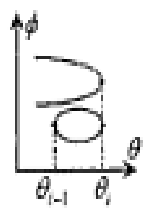

(a)

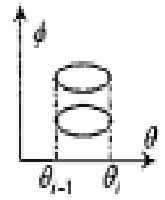

(b)
Figure 5. Crtterion 4 corresponds to the dead 


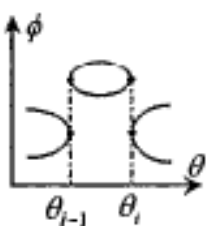

(a)

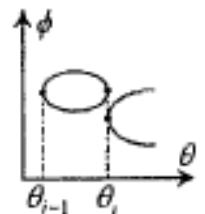

(b)

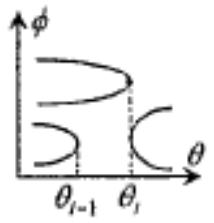

(c)

Figure 6. Criterion 5 corresponds to the dead

\section{Branch of Automatic Identification}

The first step is to define institutions branch, dead point and the input component of two adjacent stationary position data structure

As follows:

class CBranch:public CObject

\{

public:

int $\mathrm{BNo}$;

-.....

\};

class CDeadPoint:public CObject

\{

public:

double IAngle;

int ONum;

int $\mathrm{BNo}[1]$;

BOOL NOrO;

......

\};

class GDPSpace:public GObject

\{

public:

double Angle[1];

int BraNum;

int $\mathrm{BraNo}[2]$;

\}

The second step is to define three list BL, DPL and DPSL to store the information should be recorded, including BL data elements as the pointer to the class object GBranch, storage identified branch not combine with other branches of the branch information; DPL data elements for the pointer to the class object CDeadPoint, store all the dead center of information; DPSL data elements for the pointer to the class object GDPSpace, storage input component branch information between adjacent stationary position [9].

Automatic identification of branch steps are as follows:

(1) to calculate the number of input 20 kick-out output solution $\mathrm{m}$. If the foot $=4$, set up four branches $\varphi 1 \leq \varphi 2 \leq \varphi 3 \leq \varphi 4$, corresponding branch number in the order $1,2,3,4$, if $m=2$, set up two branches $\varphi 1 \leq \varphi 2$ corresponding branch number in the order 1, 2; Create $\mathrm{m}$ CBranch pointer object * $\mathrm{B} 1, \ldots, \mathrm{Bm}$, in order to add it to the BL longing tail; If $\mathrm{m}=0$, said no branch at this time. Will this as a starting point, namely the zeroth stationary position [10]. 
(2)The dry input artifacts of the ith $(I=1,2, \ldots, n)$ in a stationary position, first calculate the input theta I corresponds to the output and the number of numerical solution, and then based on the type of theta I place corresponding dead point, judge theta I executed point type, and then execute the corresponding algorithm.

(3)Repeat step (2), until you calculate all of the rest position; Then turn to the next step.

\section{Conclusions}

This paper proposes a new method of recognition space loop of monocyclic mechanism and its defects - dead point method. First by the input output equation of four times a yuan discriminant $\Delta=0$ of the input link of institutions all rest position theta $I(I=1,2, \ldots, n)$, theta $I$ put to death in the comprehensive point distribution of all types of cases, given to these types of computer automatic judgment and processing method, so as to identify the dead point position in each branch; Finally put forward to combine branch into the loop algorithm, realize the automatic recognition and defects in the loop. For the further study of space ring structure is of important theoretical value.

\section{References}

[1] Li Xuegang, Hang Yongqiang, Feng Liyan: A Sufficient and Necessary Condition for Existence of Double Crank of Planar Five Bar Linkage Studies, Vol.24 (2005).1.

[2] McCarthy J M, SohG S: Geometric Design of Linkages (Springer Verlag, America 2010).

[3] Han Jianyou, Yang Tong, etc: Linkage modern integrated theory and method/parsing theory solution domain method and the software system (higher education press, China 2013).

[4] Luck K, Modler K H. Getriebetec: Analyse-Synthese-Optimierun (Akademie Verlag, Berlin 1990).

[5] Yang Tong, Han Jianyou, Yin Lairong: Unified Synthesis Method Based on Solution Regions for Four Finitely Separated and Mixed "Point-Order" Position, Vol..46 (2011) No. 11.

[6] Yang Tong, Han Jianyou: Research on Rigid-Body Guidance Mechanism Synthesis Through Four Mixed Positions, Vol .42 (2011) No. 3.

[7] Ting K L, Wang Jun, Xue Changyu: Full Rotatability and Singularity of Six Bar and Geared Five Bar Likages, Vol 1(2010)No.2.

[8] Ting K L, Wang Jun, Xue Changyu: Full Rotatability and Singularity of Six-Bar and Geared Five-Bar Likages, Vol.2 (2010) No.1.

[9] Amir Rezaei, Alireza. Position: Jacobin and Workspace Analysis of a 3-PSP Spatial Parallelmanipulator, Vol.29 (2013) No.4.

[10] David E. Foster, Gordon R. Pennock: A Study on Kinematics and Dynamics of 3-DOF Parallel Machine, Vol.46 (2011) No.9. 